Vol. LXV 2014

\title{
HUMAN SIMULATION IN THE ASSEMBLING AND DISASSEMBLING ACTIVITIES
}

\author{
GHINEA RARES ADRIAN \\ Faculty of Machine Building/Department of Design Engineering and Robotics, "Technical University of Cluj \\ Napoca", Cluj - Napoca, Romania, rares.ghinea@muri.utcluj.ro \\ POPISTER FLORIN \\ Faculty of Machine Building/Department of Design Engineering and Robotics, "Technical University of Cluj \\ Napoca", Cluj - Napoca, Romania, florin.popister@muri.utcluj.ro \\ KISS ROBERT \\ Faculty of Machine Building/Department of Design Engineering and Robotics, "Technical University of Cluj \\ Napoca", Cluj - Napoca, Romania, robert.kiss@muri.utcluj.ro
}

\begin{abstract}
The present paper proposes a simulation algorithm of the human operator in the assembly and disassembly operations. It is presented a methodology of simulation of the human operator's in the virtual environment as well as the steps to allow identification of the variables of the simulation for an operation of assembly/disassembly/maintenance of a pneumatic valve. The time period of the tasks used in the simulation carried out in Delmia V5 are based on the time period that a real operator requires in order to complete these same tasks.
\end{abstract}

Key words: assembly, disassembly, Delmia V5, simulation, human operator

\section{Introduction}

In an impetuous worldwide market the companies from the manufacturing industries have to grant a high degree of importance to the aspects regarding the assembly strategies and methods. In order to fulfil the requirements of customers or short development lifecycle of physical models the virtual assembly processes based on computer-aided-design (CAD) models are used to obtain competitive and profitable products with low production costs through efficient assembly systems before starting-up the real production. (Ming et. al. 2013), (Liu, et. al. 2011).

The assembly process through virtual simulation which presume accomplishment of different activities (Battini, Faccio et al. 2011), such as assembly path planning sequence, assembly analysis, assembly simulation process or even operation training it became in the $20-21^{\text {th }}$ century an important research tool and concurrently a critical step in terms of product development (Yang et. al., 2013), (Leon Žlajpah, 2008),(Di Gironimo, Mozzillo et al. 2013).

All the above mentioned aspects concerning virtual assembly process simulation it can be realised nowadays with dedicated software's. In this direction using such kinds of computer applications as the one from Siemens PLM Software it can be obtained benefits regarding full traceability of products from their initial design to final manufacturing and/or optimize manual assembly operations using human simulation, automatic validation of assembly/disassembly processes, simulate and validate the flexibility of the assembly lines before actual production (w1).

Another powerful and complete solution that can be used as a tool in defining, creating and simulate the assembly process is Delmia V5(Neamţu, Hurgoiu et al. 2012). Delmia Assembly Process Simulation (APS) offers the possibility to the users to perform activities like: assembly feasibility studies; identify the issues that appear; create and optimize the entire assembly process by defining the assembly trajectories, scenarios or even the probe points for analysis during the simulation process in an interactively approach in correspondence with the manufacturing circumstances.(w2)

In this paper the authors present a simulation algorithm concerning the human operator in assembling and disassembling operations. The developed simulation algorithm of the virtual environment, steps that carry 
to identify the variables of the simulation process of assembly/disassembly/maintenance of a pneumatic valve are realised with the help of the Delmia V5 software. The tasks created after the division of the entire assembly operations and the time frame required to complete these tasks are identical to the ones used in real working conditions in assembly work stations.

\section{Proposed algorithm}

The algorithm presented below is a general one and it can be applied to the assembly process of any kind of components.

The first stage of the algorithm is composed of the preliminary operations that involve modelling the components and creating the assembly of the studied object. In this stage all the components will be modelled in great detail so that they can be used in analysing and simulating the assembly operation. Also in this stage the components of the assembly workstation will be modelled and assembled.

Figure 1. Simulation algorithm

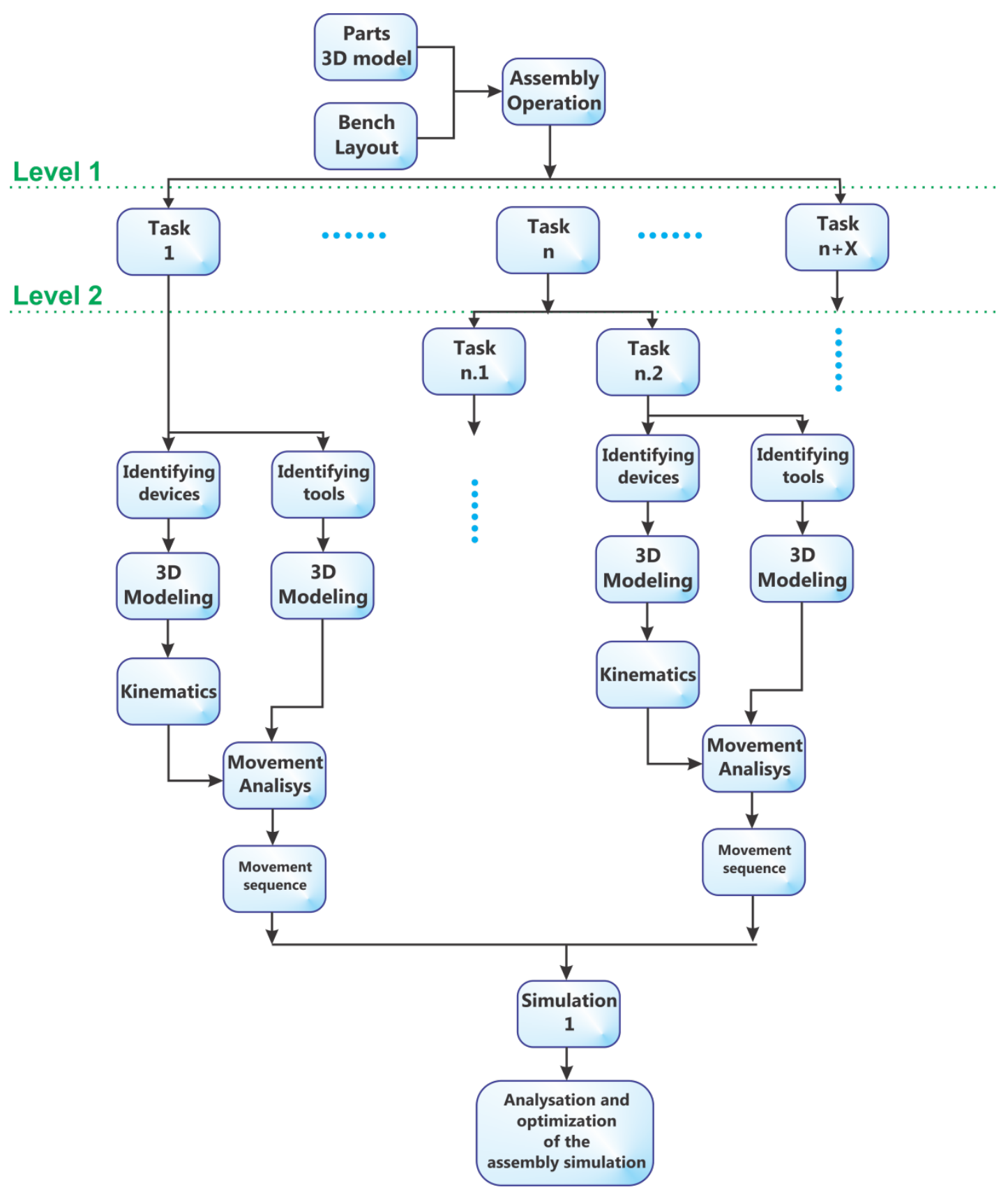

The second stage of the proposed algorithm requires dividing the assembly operation into tasks. These tasks can be grouped into several levels of complexity depending on the component or subassembly that has to be mounted in that task. Figure 1 presents tow levels of complexity corresponding with the assembly of simple components and subassemblies. Tasks can be defined only 
based on the 3D model of the assembly and the 3d model of the subassemblies of the product, which were modelled in the previous stage.

The third stage of the algorithm is the one that analysis each and every task in order to establish the necessary instruments (hand tools) and devices needed to carry out that task and also the analysis of the movement of the human operator needed for that task. Thus the first step of this phase is to identify the devices and instruments (tools) necessary for the task, then they are modelled using 3D modelling software and then they are prepared in terms of their kinematics in order to properly simulate that task. The 3D modelling and kinematics need to be done according to the supplier's datasheet. This aspect is very important in order to assure that the instruments (hand tools) are functioning as well as the ones used in real workshops.

The next step of this stage requires the analysis of the necessary movements and establishing the movement sequence. The analysis involves the identification of all types of movements that have to be made by the human operator. From the movement type's point of view these can be: translation, rotation, gripping, and ungripping. Based on these main categories there are also some types of multi-motions resulted from the combination of the four mentioned above.

The fourth stage of the proposed algorithm involves the development of the simulation for the tasks defined in the previous stage. The simulation is done independently for every task, thus at the end of this stage the first version of the assembly simulation is completed.

This version of the simulation is taken into the fifth stage of the algorithm which involves the optimisation of the assembly process based on the analysis of the simulation's results. The optimisation can be achieved through the results and the time duration obtained after running the simulation as well as the ergonomics of the assembly operations or the ergonomics of the workbench.

\section{Case study}

The case study presented in the present paper was employed in order to apply the proposed algorithm described in the previous chapter for virtual simulation of an assembly/disassembly/maintenance process.

The input data of the entire virtual assembly operations are represented by the 3D models of the parts see figure 2

The pneumatic valve is composed from elements as follows:

- Housing

- Two pressure bearings

- Two INA RST06TN bearings

- Circular spring

- Two helical springs

- Cylinder with guiding groves

- Rotation cylinder to which it is connected the valve

- Sealing cap

- One pin

- Connecting element between valve and the tap

Figure 2. 3D model of the pneumatic valve and real components

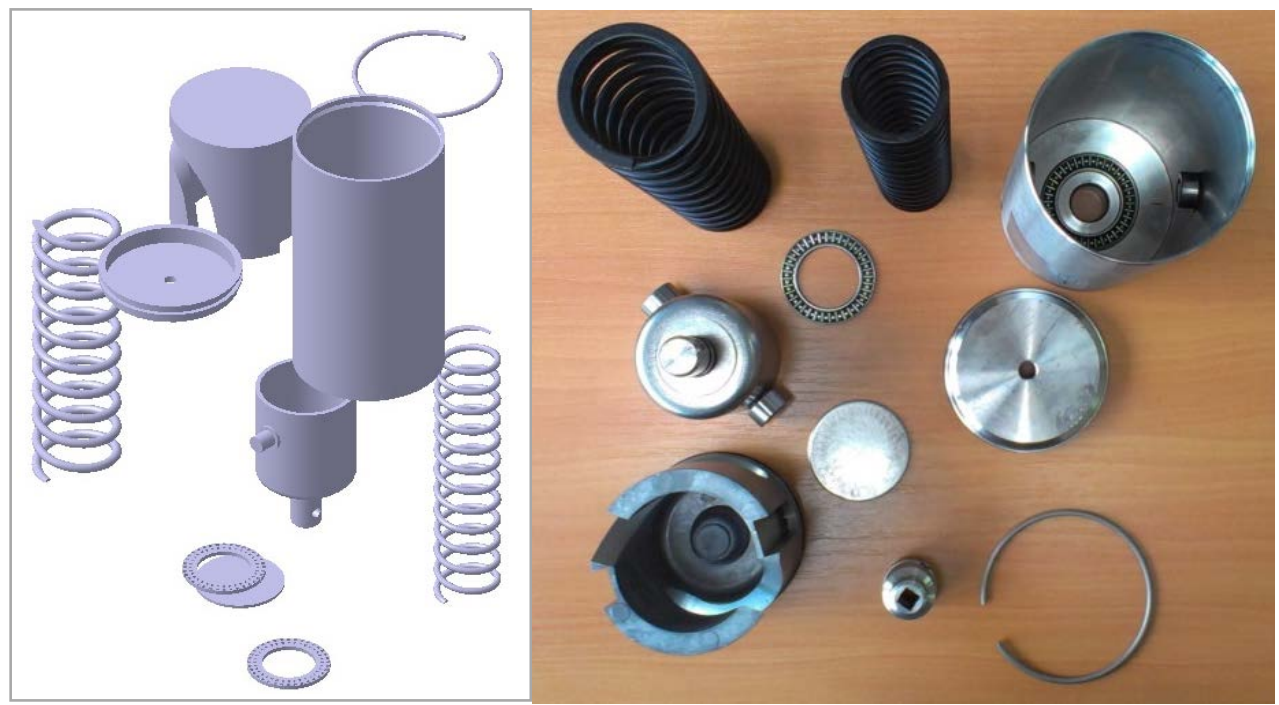




\subsection{Stage 1: Modelling the components and the assembly workstation}

In this stage, besides the components of the pneumatic valve, also the workstation was modelled as shown in figure 3. The working bench, within the virtual simulation process where the assembly/disassembly activities are to be simulated was design correspondent with the one used in real time in the workshop.

Figure 3. 3D model of the work bench layout

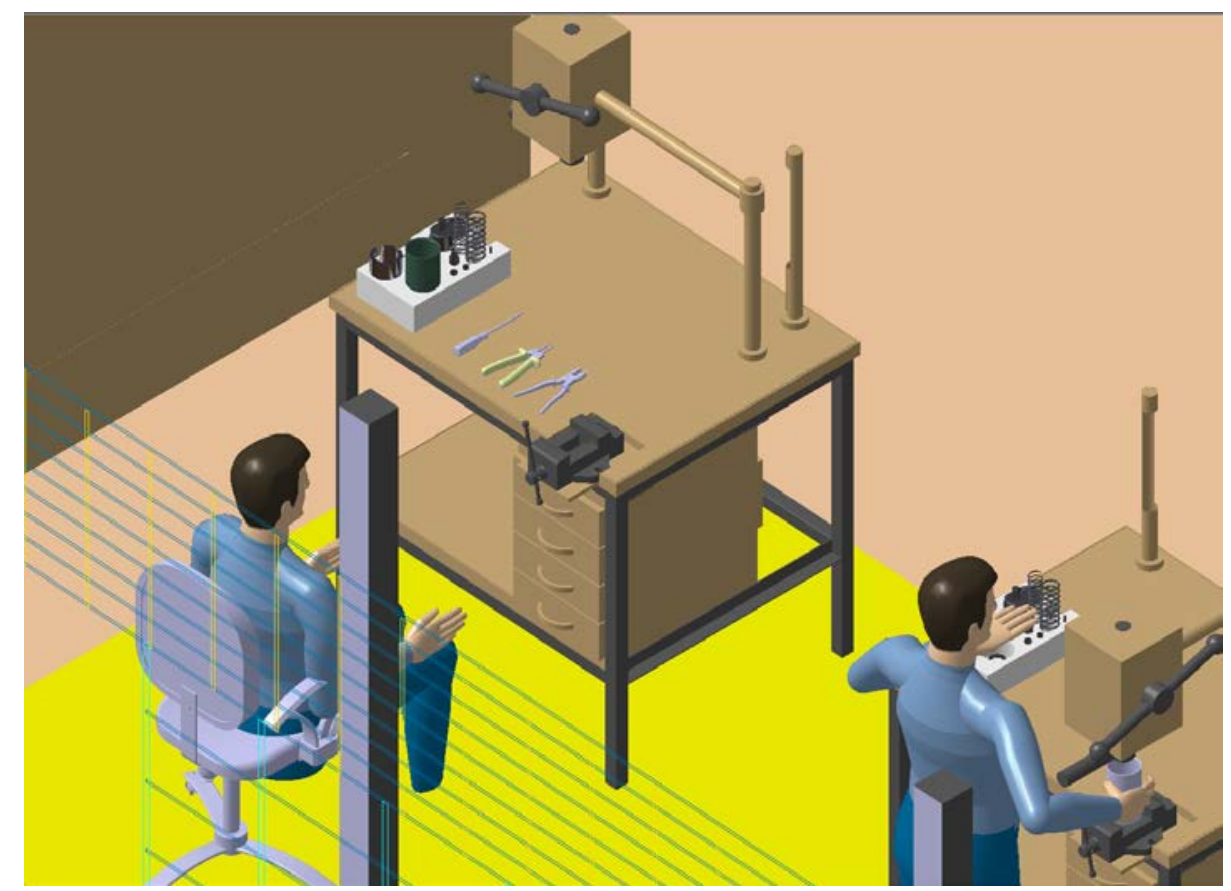

\subsection{Stage 2: Assembly tasks}

In the second stage were defined the tasks necessary to accomplish the assembly operation. Thus the authors have identified ten different tasks (Figure 4) that were arranged as shown in Figure 5.

Figure 4. The tasks defined for the assembly operation

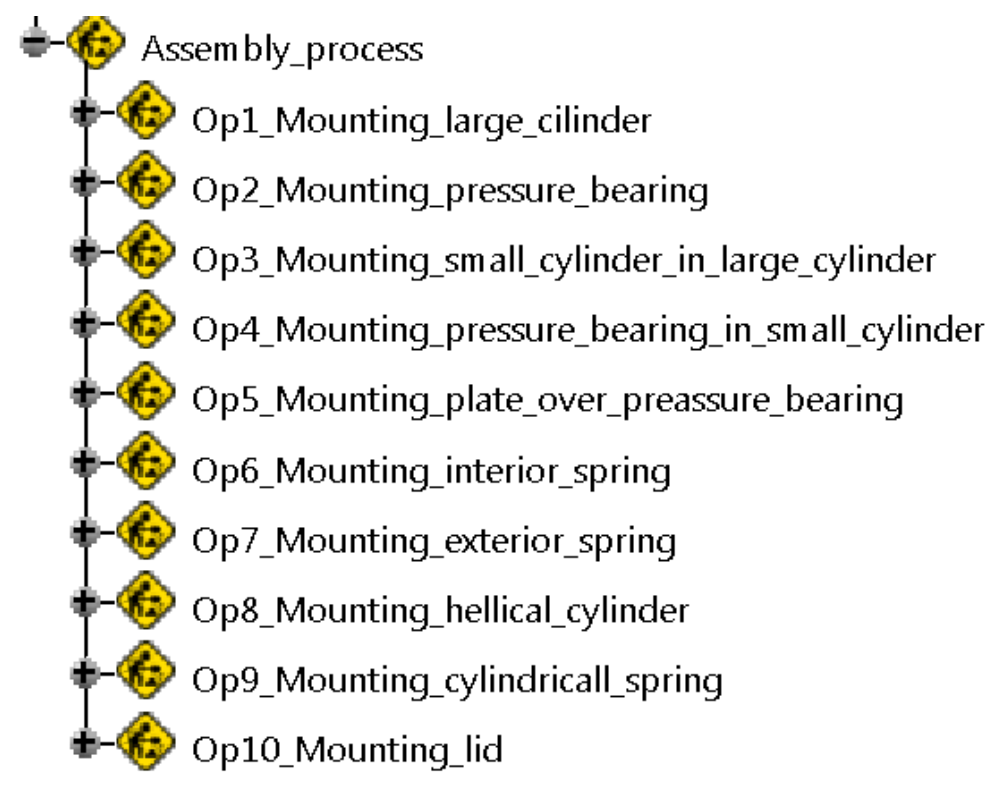

Figure 5. Tasks defined for the assembly process in Delmia V5

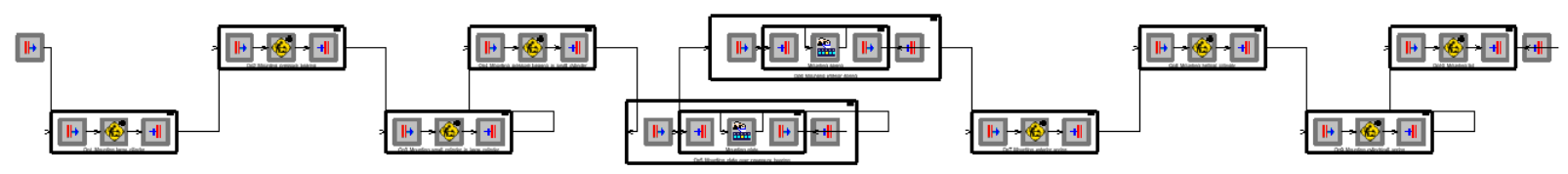




\subsection{Stage 3: Task analysis}

After analysing the ten tasks instruments and hand tools that are needed for the assembly were identified: compression device for the two springs and the following hand tools: screwdriver, pliers and a special plier for springs. They were modelled and placed on the work bench as shown in Figure 3.

Figure 6. Analysis of the motion necessary to create a task

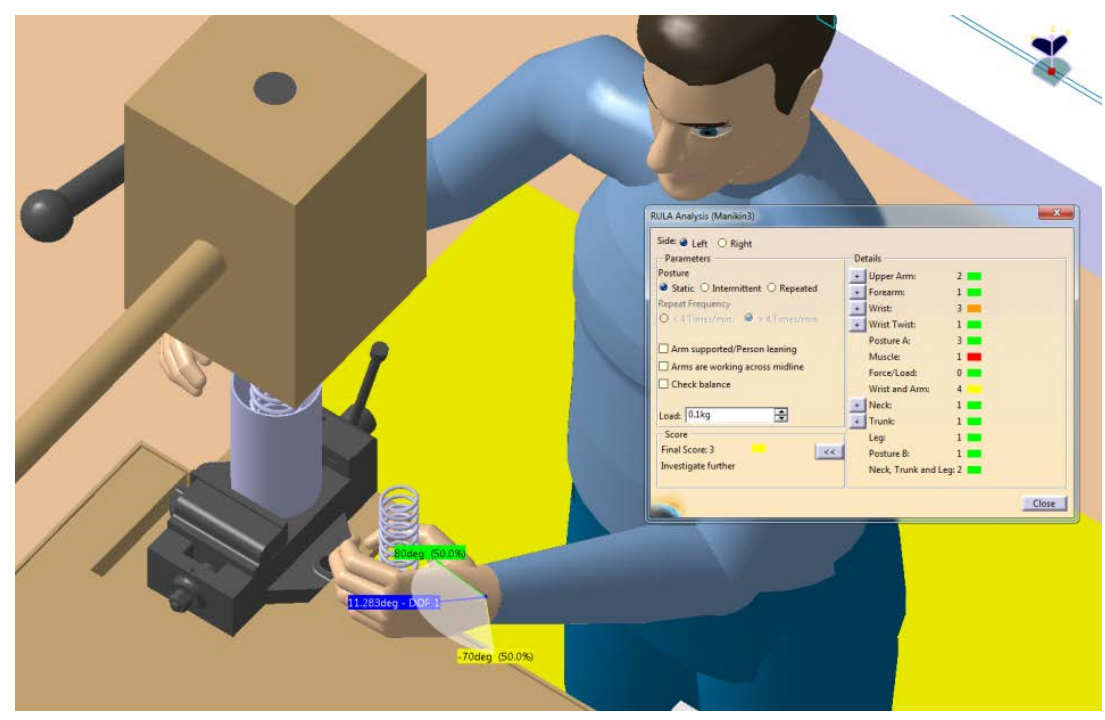

After completely assembling the workbench the next step is the motion analysis (Figure 6) and then to create the motion sequences required to create a task. Figure 7 presents the trajectories on which the the components will be inserted in the final assembly.

Figure 7. Movement sequence

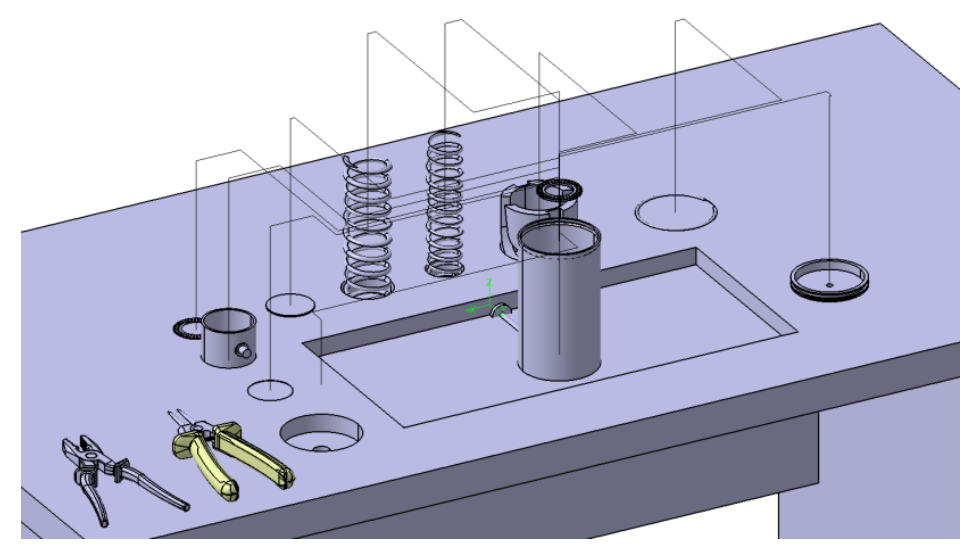

\subsection{Simulating the assembly process}

In this stage the assembly process is simulated using a virtual operator. For each task the necessary resource is associated and the required time period is defined. At this stage the time period inserted into the simulation is the same as the measured time period for the classical assembly operation. Figure 8 presents a detailed diagram of the assembly operations.

The first version of the simulation obtained based on the real process will enter the optimization stage of the assembly process (the fifth stage of the proposed model). 
Figure 8. Movement sequences

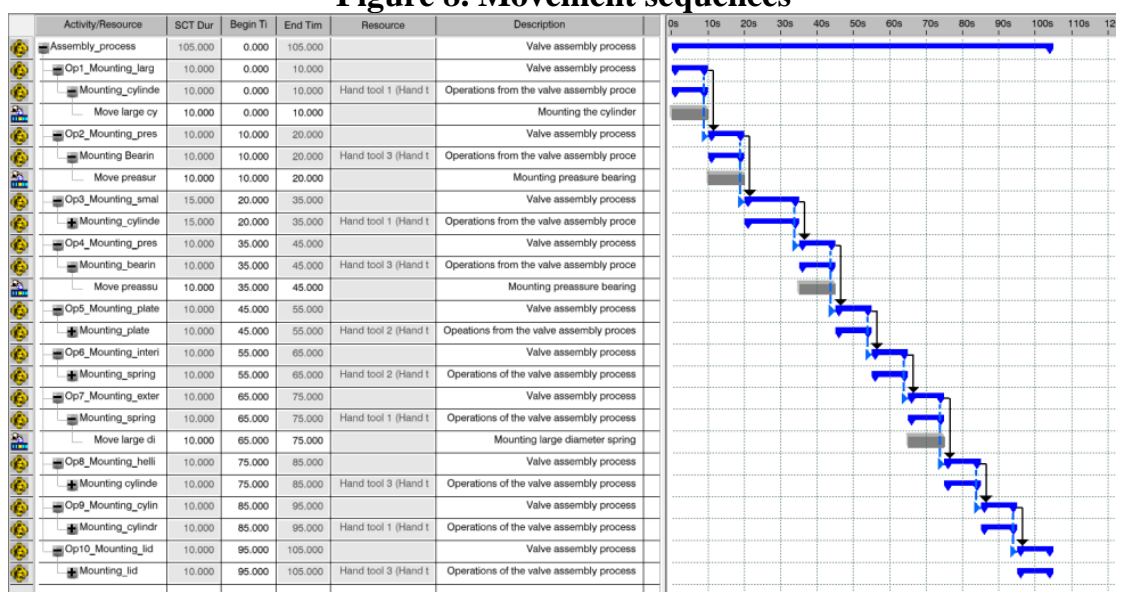

\section{Conclusions}

The simulation algorithm of an assembly process presented by the authors is generally valid for the manual assembly processes of products. The algorithm was validated through a case study aimed at assembling a pneumatic valve. The modelling and the assembly of the components and the stages of planning and the simulation of the assembly operations were performed using the DELMIA V5 software. The simulation obtained after following the five stages of the proposed algorithm will be the basis for the optimization process.

\section{References}

- Battini, D., M. Faccio, A. Persona and F. Sgarbossa (2011). "New methodological framework to improve productivity and ergonomics in assembly system design." International Journal of Industrial Ergonomics Volume: 41 Issue:(1) Pages: 30-42 ISSN: 01698141 (ISSN).

- Di Gironimo, G., R. Mozzillo and A. Tarallo (2013). "From virtual reality to web-based multimedia maintenance manuals." International Journal on Interactive Design and Manufacturing Volume: 7 Issue:(3) Pages: 183-190 ISSN: 19552513 (ISSN).

- Neamţu, C., D. Hurgoiu, S. Popescu, M. Dragomir and H. Osanna (2012). "Training in coordinate measurement using 3D virtual instruments." Measurement: Journal of the International Measurement Confederation Volume: 45 Issue:(10) Pages: 2346-2358.

- Liu, X., Liu, Y., Qi, L. (2011).Virtual assembly and simulation of vibration sieve based on a human-interface environment (Conference Paper). Procedia Engineering. Volume 15, 2011, Pages 2988-2992. ISSN:1877-7058

- $\quad$ Ming C. Leu, Hoda A. ElMaraghy, Nee A.Y.C, Ong S.K, Lanzetta M., Putz M., Zhu W., Bernard A. (2013). CAD model based virtual assembly simulation, planning and training. CIRP Annals Manufacturing Technology, Volume 62, Issue 2, 2013, Pages 799-822, ISSN: 0007-8506

- $\quad$ Yang Q., Wu D.L., Zhu H.M., Bao J.S., Wei Z.H. (2013). Assembly operation process planning by mapping a virtual assembly simulation to real operation. Computers in Industry, Vol. 64, Issues 7, (September 2013) Pages 869-879, ISSN: 0166-3615

- Žlajpah L. (2008). Simulation in robotics. Mathematics and Computers in Simulation, Vol.79, No.4, (December 2008) Pages 879-897, ISSN: 0378-4754

- w1 - Tecnomatix Assembly Planning and Validation, Optimize Manufacturing Productivity with Assembly Process Planning, accessed 2013. https://www.plm.automation.siemens.com/en_us/products/tecnomatix/assembly_planning/index.s html\#lightview-close

- w2 - Assembly Process Simulation, Datasheet. Delmia. Dassault Systems, accessed February 2013. http://www2.3ds.com/fileadmin/PRODUCTS/DELMIA/PDF/DM-12857-AssemblyProcess-Simulation-Datasheet-HR.pdf 